\title{
Factores de protección de caries dental y su efectividad de aplicación, en los menores de 6 a 9 años: Resultados de estudio piloto.
}

Factors of protection of dental caries and its application products, in children under 6 to 9 years old: Results of a pilot study.

María Isabel Romero Vásquez. ${ }^{1}$, Holguer Estuardo Romero Urrea. ${ }^{2}$

\begin{abstract}
.
DOI: https://doi.org/10.33262/cienciadigital.v9i2.376
\end{abstract}

Introduction: According to data published by the World Health Organization, 60\% $-90 \%$ of school children have dental caries worldwide. To make specific changes, we must intervene in ages susceptible to changes, such as the 6 to 12 year stage. Objective: to evaluate the effectiveness of the application of the factors of protection of dental caries in children under 6 to 9 years. Materials and Methods: Quantitative, documentary, longitudinal and experimental research was carried out in a heterogeneous sample of 60 children, who formed two groups: experimental and control, an initial diagnosis was made to the two groups, their results constitute the baseline to the experimental procedures were carried out prophylaxis, topical application of fluoride and sealants, with risk criteria, and the control was not exposed, in the second intervention is a final evaluation. For the collection of data, an instrument validated by expert judgment is used. Results: There was significant variation in the decrease of bacterial plaque indexes, dental calculus, gingivitis, dental caries and proportion of sealed molars. Conclusions: The application of protective factors is effective in controlling the risk factors and prevalence of dental caries, improving levels of oral hygiene, as well as the proportion of permanent molars sealed in the minors evaluated. Once validated, the instrument will be applied to the entire sample.

Keywords: Dental Caries, Children, Factors, Dental Plaque, Dental Sealants.

\footnotetext{
${ }^{1}$ Universidad Estatal de Milagro. Maestría en Salud Pública. Milagro, Ecuador. mromerov4@unemi.edu.ec

${ }^{2}$ Universidad Estatal de Milagro. Maestría en Salud Pública. Milagro, Ecuador. hromerou@unemi.edu.ec
} 


\section{Resumen.}

Introducción: De acuerdo a datos publicados por la Organización Mundial de la Salud 60\%-90\% de los escolares tienen caries dental a nivel mundial. Para efectuar cambios concretos hay que intervenir en edades susceptibles a cambios, como es la etapa de 6 a 12 años. Objetivo: evaluar la efectividad de la aplicación de los factores de protección de la caries dental en los menores de 6 a 9 años. Materiales y Métodos: investigación de tipo cuantitativa, documental, longitudinal y experimental, se llevó a cabo en una muestra heterogénea de 60 menores, que conformaron dos grupos: experimental y control, se realiza un diagnóstico inicial a los dos grupos, sus resultados constituyen la línea base al experimental se efectuaron procedimientos de profilaxis, aplicación tópica de flúor y sellantes, con criterios de riesgo, y el de control no estuvo expuesto, en la segunda intervención se realiza una evaluación final. Para la recolección de datos se usa instrumento validado por juicio de expertos. Resultados: Hubo variación significativa en la disminución de índices de placa bacteriana, cálculo dental, gingivitis, caries dental y proporción de molares sellados. Conclusiones: La aplicación de factores de protección es efectiva en el control de los factores riesgo y prevalencia de caries dental, mejorando niveles de higiene oral, así como proporción de molares permanentes sellados en los menores evaluados. Una vez validado el instrumento se aplicará a la totalidad de la muestra.

Palabras claves: Caries dental, niños, factores, placa bacteriana, sellante dental.

\section{Introducción.}

Los ácidos que resultan de la acción de microorganismos sobre los hidratos de carbono provocan la caries dental, es decir, esta patología se inicia cuando la interrelación entre los microorganismos y su retención en la superficie dentaria, porque los productos metabólicos desmineralizantes ácidos alcanzan una alta concentración en la placa bacteriana, por aporte excesivo de azúcares en la alimentación (Barrancos Mooney Julio, Barrancos Patricio, 2008). De acuerdo a las estadísticas es una de las enfermedades de mayor prevalencia en los niños (Selwitz Robert, Amid Ismail, Nigel Pitts, 2006) (Martins Paiva Saul et al, 2014), y es uno de los principales problemas de salud pública a nivel mundial (Seif Tomás, 1997). Por lo que, se han intensificado las actividades encaminadas a la prevención de esta enfermedad, a través de la aplicación de factores de protección.

De acuerdo con la información publicada de la Organización Mundial de la Salud (OMS) El 60\%-90\% de los escolares tienen caries dental en todo el mundo (OMS, 2012) (GranthamMcGregor Sally ET AL, 2007). El tratamiento de enfermedades dentales consume del 5\% al $10 \%$ de los presupuestos sanitarios en los países ricos. La caries dental no se suele tratar en países de ingresos más bajos, donde su costo excedería todos los recursos financieros disponibles para la atención de la salud infantil (OMS, 2015), por lo que podría convertirse 
en una pandemia produciendo a su vez, otras afecciones crónicas que complicarían la salud integral de los menores. Existe información relacionada a la prevalencia de caries dental en Ecuador correspondiente a junio del 2014, donde se encontró que en niños de 3 a 11 años de edad existe una prevalencia de 62,39\% y en individuos de 12 a 19 años de edad una prevalencia de 31,28\% (Martins Paiva Saul et al, 2014) (Raza Ximena et al, 2011). Esto define un nivel severo de acuerdo con lo establecido por la OPS/OMS (MSP, 2015). Así mismo, la OMS en el año 2000, propuso que: la promoción de la Salud bucodental debe ser prioritaria en las poblaciones de niños preescolares y escolares (OMS, 1989). El Ministerio de Salud Pública (MSP) del Ecuador, dentro del componente de las prestaciones de servicios odontológicos, en cuanto a la atención preventiva, ejecuta a nivel nacional el Programa Nacional de "Prevención de la Caries Dental", que tiene el propósito de disminuir la prevalencia de la caries dental, mediante la aplicación de factores de protección (MSP, 2009), a partir del 2017 se intensifican las acciones con la ejecución de la Campaña "Ecuador Sin Caries", con enfoque preventivo, pragmático y de atención prioritaria, dirigida a dar tratamiento oportuno a los primeros molares definitivos de los menores entre 6 y 9 años. Sin embargo, dentro del grupo etareo de 6 a 9 años (MSP, 2010), que acudió al Centro de Salud Los Pinos, ubicado en la ciudad de Milagro, en el periodo de enero a junio del 2018, 7 de cada 10 menores presentan caries dental.

Este estudio es de vital importancia, porque se analiza la eficacia de un Programa del Ministerio de Salud Pública (MSP) del Ecuador, el mismo que tiene un elevado costo de los tratamientos odontológicos, dirigido a un grupo etareo específico, y que puede evitarse asegurando la efectividad de la ejecución. El resultado hará posible establecer la implementación de una estrategia de calidad para el mejoramiento de la ejecución de medidas preventivas para la prevalencia de ésta patología bucal.

El propósito de la investigación es evaluar la efectividad de la aplicación de los factores de protección de caries dental, a través de la descripción de los índices de placa bacteriana, cálculo dental y gingivitis; la comparación del número de piezas dentales cariadas y pérdidas desde la línea base hasta un control posterior a la aplicación de los factores de protección de la caries dental. Y finalmente se revisará el estado actual de las piezas dentales que recibieron sellantes, los resultados servirán para evidenciar las necesidades reales de la población infantil del sector de estudio.

\section{Factores de riesgo de caries dental}

Se señala como causa principal a la placa bacteriana (Wolff Mark Steven y Larson Charlie, 2009). La placa bacteriana mineralizada que se forma sobre la superficie de los dientes naturales y las prótesis dentales se denomina cálculo dental (Newman Michael, Takei Henry y Klokkevold Perry, 2010). El cálculo dental indica las consecuencias de una deficiente higiene oral. En otras palabras, la placa bacteriana surge como consecuencia de una mala higiene bucal (Roche Martínez Alina ET AL, 2009). La gingivitis que es la inflamación 
gingival, es más común en niños y adolescentes, una de las causas fundamentales es la presencia de la placa bacteriana (Hernández Daniela et al, 2013). Por lo tanto, el factor de riesgo primordial para que el menor presente caries es una higiene oral deficiente (Hernández Marín, 2013).

\section{Edades adecuadas para iniciar la prevención}

La resolución de las patologías buco-dentales, más prevalentes en menores en edad escolar, deben ser el principal propósito del odontólogo (Henostrosa Haro, Gilberto, 2007). Al ser la etiología de la caries de naturaleza multifactorial, su tratamiento requiere la implementación de estrategias tanto de educación para la higiene, como orientación nutricional (SIGN, 2014). Ya que frecuentemente comienza durante la niñez y a menudo tiene secuelas para toda la vida, la odontología preventiva debe comenzar tempranamente en la vida (Nowak Arthur, Casamassimo Paul, 2002). Para lograr los resultados concretos a través de la promoción hay que actuar en edades susceptibles a los cambios (Rowan-Legg Anne, Canadian Paediatric Society, Community Paediatrics Committee, 2013). Si en el período de 6 a 12 años se desarrolla el aprendizaje imitativo de la conducta y se comienza a tomar conciencia de lo que es bueno o malo, se considera el momento idóneo para el desarrollo de comportamientos saludables y donde el impacto sería mayor (Dueñas Becerra Jesús, 1999).

Aproximadamente a los 6 años, por detrás de los molares de leche, tanto arriba como abajo, salen los primeros molares definitivos o molares de los 6 años. Por esta razón necesitan una atención especial, porque puede cariarse muy pronto y confundirlo con una muela de leche y perderla de forma temprana (MSP, 2010), siendo ésta la edad más oportuna para aplicar medidas preventivas como la práctica del cepillado dental (Hernández Daniela et al, 2013), que permitirá la remoción integral diaria de la placa (Mc Donald, Ralph y Avery, 2018).

\section{Factores de protección}

De acuerdo a los protocolos odontológicos de promoción y educación del MSP, la enseñanza en higiene bucal debe brindarse de acuerdo a los diferentes ciclos de vida. Y los factores de protección de la caries dental incluyen: profilaxis dental o limpieza dental profesional, topicación con flúor gel y aplicación de sellantes de fosas y fisuras (MSP, 2013).

La profilaxis dental es el procedimiento odontológico que remueve la placa bacteriana blanda o mineralizada (cálculo dental) (Chávez Mario, 1996), se lo realiza mediante el raspaje con instrumentos manuales y ultrasonido, después el pulido las superficies dentales con cepillo y copa de caucho rotatoria, utilizando pasta abrasiva (MSP, 2013).

La aplicación de fluoruros de forma tópica permite la aparición y control de la caries y su detención en procesos agudos (Cardozo Beatriz Juana, 2016), facilita la incorporación de calcio a los tejidos duros del diente y a su remineralización (Sanchez Alberto, Childers, Fox, 
Bradley, 1997). Se necesitan medidas de salud pública para el uso adecuado, de modo de evitar el deterioro dental (OMS, 2018).

Se realiza posterior a la profilaxis dental, se secan los dientes, para permitir la mayor absorción de flúor, se introduce una cubeta con contenido de flúor en gel al $2 \%$ en la boca del paciente por cuatro minutos, presionándola contra los dientes, para asegurar el cubrimiento de los espacios interproximales y se le pide al paciente que cierre la boca para ayudar a llevar el fluoruro alrededor de todas las superficies dentales (MSP, 2013).

Los sellantes son una maniobra de prevención de la enfermedad para el paciente que presenta fosas y fisuras pronunciadas en el elemento dental (SIGN, 2014), técnica que modifica las áreas anatómicas más retentivas de la cara masticatoria de los dientes, mediante la utilización de diversos materiales (Nowak Arthur, Casamassimo Paul, 2002). El avance de la caries se desarrolla en forma rápida y puede evolucionar en el transcurso de 6 meses de una forma incipiente a lesiones avanzadas (Zaror Carlos; Pineda Patricia; Villegas Mónica, 2011).

Para su aplicación se lava y seca las fosas y fisuras por sellar de 10 a 15 segundos; se usará el mismo líquido del ionómero de vidrio; se lava, seca y se coloca ionómero de vidrio en pequeñas cantidades en fosas y fisuras; se coloca una pequeña capa aislante de vaselina y se realiza técnica de dígito-presión en las fosas y fisuras de las piezas por sellar; finalmente se coloca una capa extra de vaselina como aislante (MSP, 2013).

Si echamos un vistazo sobre, Axelsson Per and Jan Lindhe, en su investigación "Effect of fluoride on gingivitis and dental caries in a preventive program based on plaque control" (1975), concluyen que, la limpieza profesional quincenal, combinada con meticulosas instrucciones de cepillado daban como resultado, un alto estándar de higiene bucal. Su propósito fue evaluar un programa preventivo basado en un control meticuloso de la placa. A través de una investigación de tipo cuantitativa y experimental, examinaron 82 niños; 41 en la prueba y 41 en el grupo control (Axelsson, Per and Lindhe, Jan, 1975).

Consideramos también a, Tranaeus Sofia, Susan et al, en su investigación "Application of quantitative light induced fluorescence to monitor incipient lesions in caries-active children. A comparative study of remineralisation by fluoride varnish and professional cleaning", aportaron que, las aplicaciones repetidas de fluoruro tuvieron un efecto favorable en la remineralización de las lesiones de la mancha blanca según se midió después de 6 meses. El estudio comparó el tratamiento con barniz de flúor y la limpieza dental profesional para la remineralización de las lesiones de la mancha blanca en adolescentes con caries activa (Tranaeus Sofia et al, 2001).

Conviene distinguir a, Viaña Francys, et al; en su estudio "Efectividad de sellantes de fosas y fisuras en la prevención de caries dental en molares sanos y con fluorosis en escolares", afirmaron la efectividad de sellantes de fosas y fisuras en la prevención de la caries dental, 
al ser una terapéutica preventiva eficaz ante el riesgo de caries. El propósito de su estudio fue comprobar la efectividad de sellantes de fosas y fisuras en la prevención de caries dental en molares sanos y con fluorosis en escolares; a través de una investigación de enfoque cuantitativo bajo la modalidad de investigación de campo, siendo un estudio epidemiológico descriptivo, no experimental transversal. La muestra censal estuvo conformada por 234 niños, evaluándose un total de 770 molares (Viaña Francys, Belkis López, Mary Borges, 2005).

También cabe hacer énfasis a, Tapias Ledesma et al, en su estudio "Efectividad de los selladores de fisuras en una población infantil con alto riesgo de presentar caries", quienes sostienen que, se debe ofertar selladores oclusales en el primer molar permanente a todos los escolares, ya que los escolares sin programa preventivo y sin selladores oclusales presentan un riesgo significativamente mayor de desarrollar caries. El propósito del estudio fue conocer la efectividad de los selladores oclusales en una población infantil; a través de una investigación de cohortes prospectivo. Se estudiaron 607 escolares que se incluyen a los 6 años de edad y se siguen durante 4,5 años (Tapias Ledesma, Jiménez-García, Lamas, Gil de Miguel, 2002).

\section{Metodologia.}

La presente investigación es de enfoque cuantitativa, de - tipo documental y de campo, de corte longitudinal y de diseño experimental. Es un estudio de tipo retrospectivo tomando como fuente de información la historia clínica única de odontología (HCUO) del MSP, donde se encontraban los registros de actividades odontológicas realizados por los estomatólogos. El instrumento que se utilizó para la recolección de datos fue una Guía de Observación validada por 6 expertos-juicio de expertos, 3 técnicos y 3 metodológicos- metodólogos. Se analizó pertinencia, la validez, confiabilidad y. El puntaje de validación fue 93\%, 91\%, 92\% 92. La estructura del instrumento consta de 4 partes, correspondientes a los 4 objetivo-s respectivamente, general pregunta 1 a la 10, específico 1 pregunta 11 a la 20, específico 2 pregunta 21 a la 30 y específico 3 pregunta 31 a la 40 .

La muestra se la selecciona de los menores de 6 a 9 años que acudieron a la consulta odontológica del Centro de Salud Los Pinos, en el periodo enero a junio 2018, obteniendo como resultado la participación de 60 niños, se determina el grupo etario por los programas desarrollados en el MSP, en su estrategia Ecuador Libre de Caries1132 346. De la muestra obtenida se forman dos grupos, el uno que consta de 50 niños, a quienes se les aplicaron factores de protección de la caries dental, a quienes se les denominó grupo experimental; y se toma 10 pacientes como grupo de control, quienes no estuvieron expuestos. Por ser una experimentación directa y el análisis de los grupos representan inversión de tiempo, por la dificultad de la asistencia de los niños, se realiza un estudio piloto, con el propósito de medir la eficacia del instrumento y del proceso descrito en la investigación, para la formación del 
grupo de investigación se escogió 10 niños de cada grupo, determinados aleatoriamente según el listado de asistencia.

Se realiza un estudio diagnóstico de los grupos, al uno se aplica los protectores y al otro grupo solo se observa el proceso por un periodo de 3 meses.

Durante el desarrollo del estudio se analizó a través de un estudio exploratorio tipo diagnóstico de los dientes y su estado de salud, comprobando en cada uno de ellos: Durante el desarrollo del estudio se analizó: el indicador de placa bacteriana, cálculo dental y gingivitis, relacionado con las profilaxis odontológicas ejecutadas; el número de piezas dentales cariadas y perdidas, relacionado a las topicaciones de flúor; y se determinó el estado de las piezas dentales que recibieron sellantes, presentados con la edad en la primera intervención.

\section{Primera intervención}

Al grupo experimental se le realizó el llenado completo y correcto de la HCUO y posterior a ésta acción, se le brindó las actividades de prevención que fueron agrupadas en un paquete básico de atención odontológica preventiva. Que incluía la eliminación de la placa bacteriana blanda y mineralizada mediante la profilaxis dental, si fue necesario la aplicación tópica de flúor, fosfato acidulado al $2 \%$ por un minuto según la indicación del fabricante. La intervención también incluyó la aplicación de sellantes de fosas y fisuras en molares permanentes y temporarios de acuerdo al criterio de riesgo.

Al grupo de control, se le realizó el llenado completo y correcto de la HCUO. Con los resultados se crea la línea base.

\section{Proceso de las evaluaciones clínicas}

Se realizó la segunda intervención a los dos grupos con un rango mínimo de 3 meses con la ejecución del llenado completo y correcto de la HCUO, durante su segunda visita en el consultorio de odontología, registrando en éste documento los índices de placa bacteriana, cálculo dental, gingivitis, número de piezas dentales cariadas y perdidas y se registró el estado de las piezas dentales que recibieron sellantes en la primera intervención.

Los exámenes odontológicos se realizaron en el consultorio de odontología del Centro de Salud Los Pinos, empleando el método visual con ayuda de luz fría, un espejo bucal plano №5 y limpiando con hisopos las superficies dentarias antes de ser examinadas. Para el diagnóstico de caries dental se emplearon los criterios de la ICDAS-ICCMS357 368.

El examen de cada diente se realizó recorriendo con un explorador la superficie dentaria desde el plano oclusal hacia el margen gingival, sin el uso de sustancia reveladora. De acuerdo con el nivel de higiene oral, cada niño fue clasificado como presentando una buena $(0,0$ a 1,0$)$, regular $(1,5$ a 2,0$)$ o mala $(2,0$ a 3,0) condición de higiene oral. 
La efectividad se evaluó mediante la variación de 9 indicadores medidos antes y 3 meses después de iniciada la primera intervención: a. cambios observados en el índice de placa bacteriana; b. cambios observados en el índice de cálculo dental; c. cambios observados en el índice de gingivitis; d. cambios observados en el índice de piezas dentales cariadas permanentes; e. cambios observados en el índice de piezas dentales perdidas permanentes; $\mathrm{f}$. cambios observados en el índice de piezas dentales cariadas temporarias; g. cambios observados en el índice de piezas dentales perdidas temporarias; h. presencia de caries durante la segunda toma en las piezas dentales temporarias selladas en la primera toma; y i. presencia de caries durante la segunda toma en las piezas dentales permanentes selladas en la primera toma.

El correspondiente estudio se efectuó en el software estadístico SPSS (Statistical Package for the Social Cience - Paquete Estadístico para las Ciencias Sociales) en su versión 24. La categorización de las variables de estudio será expuesta en porcentajes, comparadas bajo el test del Chi Cuadrado de Pearson y la correlación estadística, además de utilizar para la muestra la estadística descriptiva, se evaluóará la diferencia considerada estadísticamente en el valor de $\mathrm{p}<0.05$, generando el nivel de significación válido para el estudio.

\section{Resultados.}

Los presentes resultados están basados en la evaluación longitudinal de la observación de 20 historias clínicas de menores entre 6 y 9 años de edad.

La tabla 1, presenta que la muestra correspondiente al grupo experimental refleja disminución del índice de placa bacteriana; mientras que el grupo de control presenta incremento.

Tabla 1. Cambios observados en el índice de placa bacteriana que presenta el menor por edad

\begin{tabular}{|c|c|c|c|c|c|c|}
\hline \multicolumn{2}{|c|}{} & \multicolumn{2}{c|}{ Grupo Control } & \multicolumn{2}{c|}{ Grupo Experimental } & \\
\cline { 2 - 7 } & Incrementó & $\begin{array}{c}\text { Sin } \\
\text { Variación }\end{array}$ & Disminuyó & $\begin{array}{c}\text { Sin } \\
\text { Variación }\end{array}$ & TOTAL \\
\hline \multirow{3}{*}{$\begin{array}{c}\text { Edad del } \\
\text { encuestado }\end{array}$} & 6 años & - & $10 \%$ & $10 \%$ & - & $\mathbf{1 0 \%}$ \\
\cline { 2 - 7 } & 7 años & $40 \%$ & - & $30 \%$ & $10 \%$ & $\mathbf{4 0 \%}$ \\
\cline { 2 - 7 } & 8 años & - & $20 \%$ & $20 \%$ & - & $\mathbf{2 0 \%}$ \\
\cline { 2 - 7 } & 9 años & $30 \%$ & - & $30 \%$ & - & $\mathbf{3 0 \%}$ \\
\hline \multicolumn{2}{|c|}{ Total } & $\mathbf{7 0 \%}$ & $\mathbf{3 0 \%}$ & $\mathbf{9 0 \%}$ & $\mathbf{1 0 \%}$ & $\mathbf{1 0 0 \%}$ \\
\hline
\end{tabular}

Fuente: Elaboración propia

El gráfico 1, muestra el comportamiento de los índices de placa bacteriana, cálculo y gingivitis, con una disminución en el grupo experimental, y un incremento en el de grupo de control. 
Gráfico 1. Cambios observados en el índice de higiene oral simplificada que presenta el menor

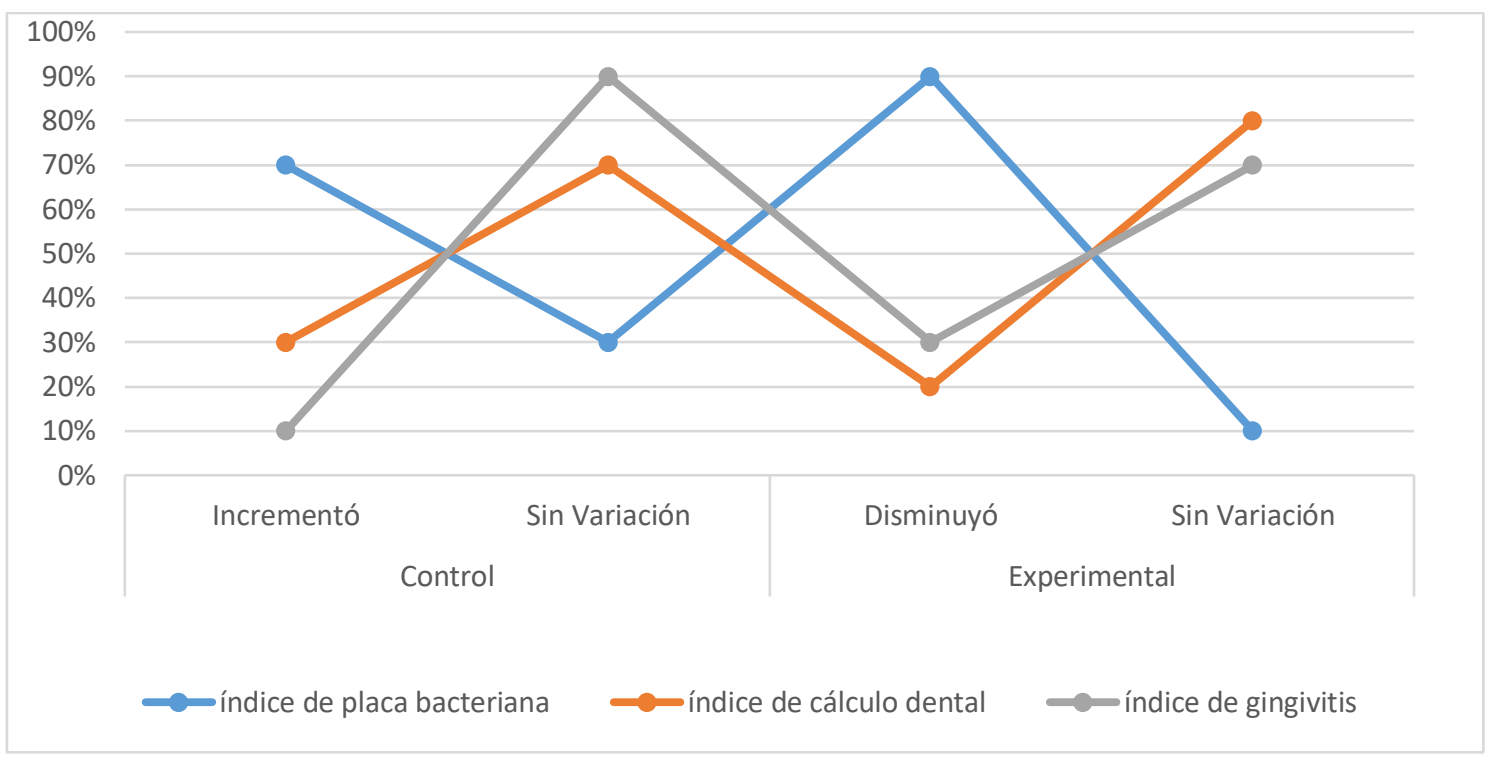

Fuente: Elaboración propia

La tabla 2, demuestra que el número de piezas dentales cariadas permanentes en el grupo experimental muestra disminución considerable con relación a la primera y segunda toma; mientras que el grupo de control no presenta variación alguna.

Tabla 2. Número de piezas dentales cariadas permanentes durante la primera y segunda toma por edad

\begin{tabular}{|c|c|c|c|c|c|c|c|c|c|c|}
\hline \multirow{8}{*}{ 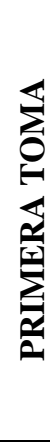 } & & & \multirow{2}{*}{\multicolumn{3}{|c|}{\begin{tabular}{|c|} 
Grupo Experimental \\
$\begin{array}{c}\text { Número de piezas dentales } \\
\text { cariadas }\end{array}$ \\
\end{tabular}}} & \multirow{2}{*}{\multicolumn{4}{|c|}{$\begin{array}{c}\text { Grupo Control } \\
\text { Número de piezas dentales cariadas } \\
\end{array}$}} & \multirow{3}{*}{ Total } \\
\hline & & & & & & & & & & \\
\hline & & & 0 & 1 & 2 & 0 & 1 & 2 & 4 & \\
\hline & \multirow{4}{*}{$\begin{array}{l}\text { Edad del } \\
\text { encuestado }\end{array}$} & 6 años & - & - & $10 \%$ & $10 \%$ & - & - & - & $10 \%$ \\
\hline & & 7 años & $10 \%$ & $20 \%$ & $10 \%$ & - & $20 \%$ & $10 \%$ & $10 \%$ & $40 \%$ \\
\hline & & 8 años & $20 \%$ & - & - & $10 \%$ & - & $10 \%$ & - & $20 \%$ \\
\hline & & 9 años & - & $10 \%$ & $20 \%$ & $10 \%$ & $10 \%$ & $10 \%$ & - & $30 \%$ \\
\hline & \multicolumn{2}{|c|}{ Total } & $30 \%$ & $30 \%$ & $40 \%$ & $30 \%$ & $30 \%$ & $30 \%$ & $10 \%$ & $100 \%$ \\
\hline \multirow{8}{*}{ 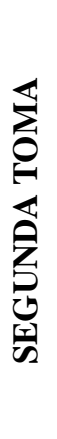 } & & & \multicolumn{3}{|c|}{ Grupo Experimental } & \multicolumn{4}{|c|}{ Grupo Control } & \\
\hline & & & \multicolumn{3}{|c|}{$\begin{array}{l}\text { Número de piezas dentales } \\
\text { cariadas }\end{array}$} & \multicolumn{4}{|c|}{ Número de piezas dentales cariadas } & Total \\
\hline & & & 0 & 1 & 2 & 0 & 1 & 2 & 4 & \\
\hline & \multirow{4}{*}{$\begin{array}{l}\text { Edad del } \\
\text { encuestado }\end{array}$} & 6 años & - & $10 \%$ & - & $10 \%$ & - & - & - & $10 \%$ \\
\hline & & 7 años & $20 \%$ & $10 \%$ & $10 \%$ & - & $20 \%$ & $10 \%$ & $10 \%$ & $40 \%$ \\
\hline & & 8 años & $20 \%$ & - & - & $10 \%$ & - & $10 \%$ & - & $20 \%$ \\
\hline & & 9 años & $20 \%$ & - & $10 \%$ & $10 \%$ & $10 \%$ & $10 \%$ & - & $30 \%$ \\
\hline & \multicolumn{2}{|c|}{ Total } & $60 \%$ & $20 \%$ & $20 \%$ & $30 \%$ & $30 \%$ & $30 \%$ & $10 \%$ & $100 \%$ \\
\hline
\end{tabular}


Fuente: Elaboración propia

La tabla 3, presenta que en el grupo experimental no hay presencia de caries en las piezas dentales permanentes que fueron selladas durante la primera toma. Mientras que el grupo de control no presenta ninguna variación.

Tabla 3. Presencia de caries durante la segunda toma en las piezas dentales permanentes selladas de la primera toma por edad

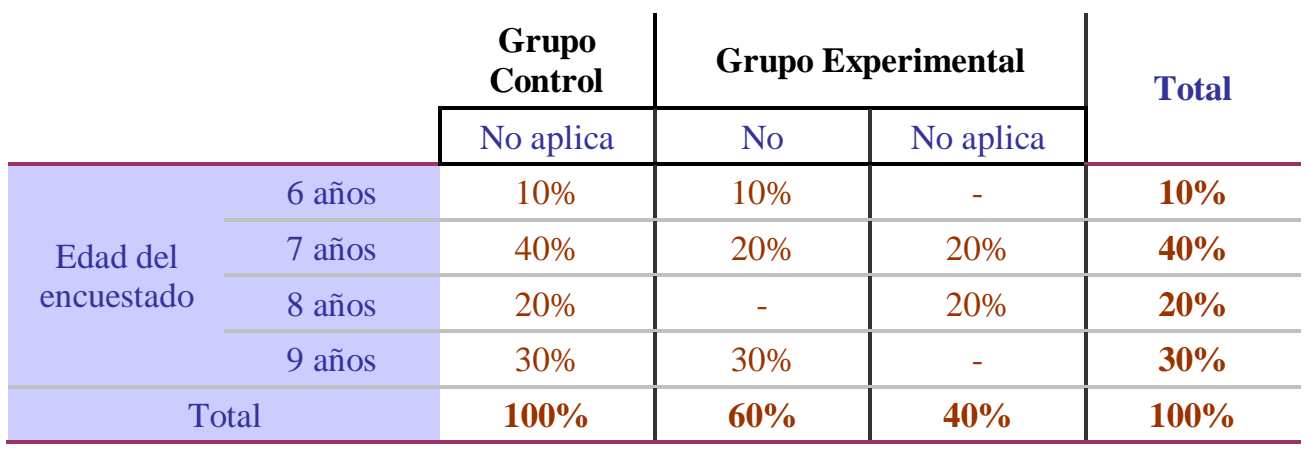

Fuente: Elaboración propia

\section{Discusión.}

Carrero Gladys et al, en su estudio, "Prevención de caries dental en primeros molares permanentes utilizando sellantes de fosas y fisuras y enjuagues bucales fluorurados", afirmaron que la aplicación de métodos combinados en la prevención de la caries dental permite potenciar sus efectos (Carrero Gladys, Fleitas Teresa, Arellano Leylan, 2006) (Martins Paiva Saul, 2014). Esta investigación realizada confirma los resultados encontrados en el estudio realizado en Ecuador, donde se demuestra la efectividad de los factores de protección de la caries dental.

En otro estudio realizado por Hanes Gales et al, se encontró que la efectividad de los sellantes se incrementó después de un seguimiento de 54 meses, alcanzando un valor de $86 \%$, comparado con el $43 \%$ obtenido en evaluaciones durante períodos de tiempo más cortos (Gale, T.J., Hanes, C.M., Myers, D.R. \& Russell, 1998) (Seif Tomás, 1997), lo que sustenta nuestra esta investigación de que son efectivos con un buen seguimiento.

Axelsson Per y Lindhe Jan, en su estudio "Effect of fluoride on gingivitis and dental caries in a preventive program based on plaque control", demuestran que la limpieza profesional quincenal de los dientes combinada con meticulosas instrucciones de cepillado daban como resultado un alto nivel de higiene bucal (Axelsson, Per and Lindhe, Jan, 1975) (OMS, 2018), en el presente estudio se demuestra que efectivamente la profilaxis odontológica ha logrado disminuir el nivel de placa en boca, dando como resultado una higiene oral buena adecuada. 
Tranaeus Sofia et al, en su estudio "Application of quantitative light induced fluorescence to monitor incipient lesions in caries-active children. A comparative study of remineralisation by fluoride varnish and professional cleaning", concluyen que las aplicaciones repetidas de fluoruro tuvieron un efecto favorable en la remineralización de las lesiones de la mancha blanca según se midió después de 6 meses (Tranaeus Sofia et al, 2001) (Zaror Carlos; Pineda Patricia; Villegas Mónica, 2011). En el presente estudio, fueron medidas en una línea base de 3 meses; sin embargo, presentaron cambios significativos en la disminución del índice de piezas dentales cariadas, debido a la remineralización de las lesiones (ICDAS, 2014).

Tapias Ledesma et al, en su estudio "Efectividad de los selladores de fisuras en una población infantil con alto riesgo de presentar caries", demuestran que los selladores oclusales en el primer molar permanente, aplicados en un grupo de alto riesgo, tienen un efecto protector frente a la caries dental (Tapias Ledesma, Jiménez-García, Lamas, Gil de Miguel, 2002). Lo que se demuestra en este experimento ya que el grupo expuesto a la aplicación de los factores de protección durante la primera toma, no presentó caries en las piezas dentales que fueron selladas dentro de la línea base.

Viaña Francys et al, "Efectividad de sellantes de fosas y fisuras en la prevención de caries dental en molares sanos y con fluorosis en escolares. Período 1999-2004", afirman que el tratamiento de sellantes de fosas y fisuras resulta ser una terapéutica preventiva eficaz ante el riesgo de caries; se evidencia por la ausencia de ésta en primeros molares permanentes con retención completa del sellante (Viaña Francys, Belkis López, Mary Borges, 2005); afirmación que sustenta este estudio, ya que una vez transcurrido los 3 meses del periodo entre la primera y segunda intervención, el sellante se encontró presente boca sobre la superficie dental aplicada, y se constató que no había presencia de caries, mostrando su efectividad y con esto hemos cumplido con la comprobación del efecto protector ante la caries dental.

\section{Conclusiones.}

- Con el presente estudio se confirma la efectividad de la aplicación de factores de protección de caries dental cuando se realizan con un seguimiento adecuado, sin considerar el criterio de riesgo, es decir, es apta para todos los menores. Por ende, se debe fortalecer los programas preventivos del MSP. Ya que, en los países desarrollados la caries dental infantil ha disminuido en las últimas décadas, debido a la implantación de programas preventivos (García Álvarez, Olga et al, 2008).

- Se debe promover la mejora del nivel de higiene oral con un control minucioso de la placa bacteriana, a través de la profilaxis odontológica; la misma que es efectiva en la disminución del índice de la misma; así como también del cálculo dental, y gingivitis, logrando el control de los factores de riesgo de caries dental. Con los resultados de la investigación se ha logrado demostrar también que la aplicación de los factores de protección nos ayuda a controlar tempranamente el proceso de desmineralización del esmalte. Por ende, se lograr reducir el número de piezas dentales cariadas. Paralelamente, se ha confirmado que una vez revisado el estado de 
las piezas dentales donde les fue aplicado los sellantes de fosas y fisuras, su estructura dental se ha conservado sana, siempre y cuando el mismo permanezca en la superficie dental. Por lo que se recomienda la aplicación de este factor de protección, haciéndolo de preferencia cuando el molar hace su erupción en boca, y tener un adecuado seguimiento.

- Para efectuar cambios concretos a través de la promoción hay que intervenir en edades susceptibles a los cambios, de acuerdo al presente estudios es efectivo intervenir a partir de los 6 años, edad donde aparece el primer molar permanente. La salud bucal infantil es un objetivo primordial en el contexto de las políticas de salud, el planeamiento preventivo es imperativo para la consecución de los programas en salud. En base a los datos obtenidos, el instrumento es válido para su aplicación a la totalidad de la muestra.

\section{Referencias bibliográficas.}

Axelsson, Per and Lindhe, Jan (1975). "Effect of fluoride on gingivitis and dental caries in a preventive program based on plaque control". Community Dentistry and Oral Epidemiology. Volumen 3. Issue 4. United States. Agosto. Pag: 156-160. Disponible en: https://doi.org/10.1111/j.1600-0528.1975.tb00300.x

Barrancos Mooney Julio (2008), Barrancos Patricio. "Operatoria Dental -Integración Clínica”. Editorial Médica Panamericana. Cuarta Edición. Buenos Aires, Argentina.

Cardozo Beatriz Juana, María Mercedes Gonzalez, Silvia Rita Pérez, Patricia Alejandra Vaculik, Elena Griselda Sanz (2015). "Epidemiología de la caries dental en niños del Jardín de Infantes "Pinocho" de la ciudad de Corrientes". Revista Facultad de Odontología ISSN $\mathrm{N}^{\circ} 1668-7280$ - Volumen DC $\mathrm{N}^{\circ} 1$ - 2016. Argentina. Disponible en: http://revistas.unne.edu.ar/index.php/rfo/article/view/1597

Carrero Gladys, Fleitas Teresa, Arellano Leylan (2006). "Prevención de caries dental en primeros molares permanentes utilizando sellantes de fosas y fisuras y enjuagues bucales fluorurados". Revista Odontológica de los Andes. Volumen 1. Mérida. Venezuela. Pag: 44-53

Chávez Mario (1996). “Odontología Sanitaria”, Tercera Edición. Publicaciones Científicas; Washington DC.

Dueñas Becerra Jesús. Educación para la salud (1999): bases psicopedagógicas. Revista Cubana Educación Médica Superior. Volumen 13 N 1 . Ciudad de la Habana, Cuba. Junio. Pag: 92-8.

Gale, T.J., Hanes, C.M., Myers, D.R. \& Russell, C.M. (1998). Performance of sealants applied to first permanent molars in a dental school setting. Pediatric

Dentistry. 20(5):341-4.

García Álvarez, Olga, y Nora M. Sexto Delgado, y Nereida Moya Padilla, y Raúl López Fernández (2008). "Intervención educativa para el desarrollo de conocimientos sobre salud bucal en la enseñanza primaria". Editorial Universidad de Ciencias Médicas de Cienfuegos. MediSur, volumen 6, $\mathrm{N}^{\circ}$. 2, Cienfuegos. Cuba. Pag: 20-24. Disponible en: https://www.redalyc.org/articulo.oa?id=180020309006 
Grantham-McGregor Sally, Yin Bun Cheung, Santiago Cueto, Paul Glewwe, Linda Richter, Barbara Strupp (2007). "Developmental potential in the first 5 years for children in developing countries". The Lancet, Volume 369. Issue 9555. Estados Unidos. Pages 60-70. ISSN 0140-6736. Disponible en: http://www.sciencedirect.com/science/article/pii/S0140673607600324

Henostrosa Haro, Gilberto (2007): “Caries Dental. Principios y procedimientos para el diagnóstico". Editorial Ripano. 1ª Edición. Universidad Peruana Cayetano Heredia. Lima. Perú. Pag: 171; 13-14.

Hernández Daniela, Compeán María, Staines María, Enzaldo Patricia. (2013). "Prevalencia de gingivitis y su relación con la higiene bucal en escolares". Revista IMBIOMED. Odontología Actual. Boletín 10(122):. México. 2013. Pag: 28-34.

Hernández Marín, C. A., González Fortes, B., Yero Mier, I., \& Rivadeneira Obregón, A. M. Caries dental y la higiene bucal en adolescentes de 12 a 15 años. Área Norte Sancti Spíritus. 2010. Gaceta Médica Espirituana, 15(1), 03-09.

ICDAS FOUNDATION (2014): "Guía de referencia rápida ICCMS ${ }^{\mathrm{TM}}$ para clínicos y educadores". Pagina Web Oficial. USA. Diciembre. Disponible en: https://www.iccms-web.com/uploads/asset/592840df43a62191555433.pdf. Extraído el 5 de enero del 2019.

ICDAS - International Caries Detection and Assessment System. (2010). Available from: https://www.sdpt.net/accesocontenidosICDAS-ICCMS.htm

Martins Paiva Saul, Evelyn Álvarez Vidigal, Jenny Abanto, Ailin Cabrera Matta,Refugio Antonio López Robles, Carla Masoli, Sonia Alejandra Echevarría Lopez, Maria Guadalupe Mongelos de Idoyada, María Elena Guerra Gamboa, Adriana Rocio Amado Schneider (2014): "Epidemiología de la caries dental en america latina". Revista de Odontopediatría Latinoamericana. Volumen 4, No. 2, Año 2014. Disponible en: https://www.revistaodontopediatria.org/ediciones/2014/2/art-4/. Consultado el: 15/01/2019.

Mc Donald, Ralph y Avery (2018). “Odontología pediátrica y del adolescente”. Editorial Médica Panamericana. Décima Edición. Buenos Aires.

Ministerio de Salud Pública. MSP. (2015). “Caries”. Guía Práctica Clínica. Primera Edición Quito: Dirección Nacional de Normatización; Disponible en: http://salud.gob.ec

Ministerio de Salud Pública. MSP. ()2010. "Estándares, Indicadores e Instrumentos Para Medir La Calidad De La Atención De Salud Bucal En Los Servicios Odontológicos Proceso De Normatización Del Sistema Nacional de Salud. Área De Salud Bucal”. Componente Normativo Atención Integral en Salud Bucal. Quito. Ecuador. Junio.

Ministerio de Salud Pública. MSP. (2010). "Manual de Educación para Salud Bucal para Maestros y Promotores". Normatización del Sistema Nacional de Salud. Área de Salud Bucal. Quito. Disponible en: https://aplicaciones.msp.gob.ec/salud/archivosdigitales/documentosDirecciones/dnn larchivos/MANUAL\%20EDUCATIVO\%20PARA\%20LA\%20SALUD\%20BUCA L\%20PARA\%20MAESTROS\%20Y\%20PROMOTORES.pdf 
Ministerio De Salud Pública (MSP) (2009): 'Normas y Procedimientos de atención en Salud Bucal - Primer Nivel". Normatización del Sistema Nacional de Salud. Área de Salud Bucal. Quito.

Ministerio de Salud Pública. MSP (2013). "Protocolos odontológicos". Primera Edición.

Programa Nacional de Genética y Dirección Nacional de Normatización. Quito. Ecuador. Disponible en: http://salud.gob.ec

Nowak Arthur, Casamassimo Paul (2002). "The dental home: A primary care oral health concept". The Journal of the American Dental Association. Volume 133. Issue 1. Estados Unidos. Pag: 93-98. ISSN 0002-8177. Disponible: http://www.sciencedirect.com/science/article/pii/S0002817714647218

Organización Mundial de la Salud. OMS (2018). "Exceso o cantidad inadecuada de flúor". Programa Internacional de Seguridad de las Sustancias Químicas. Sitio Web Oficial. Ginebra. Suiza. Disponible en: http://www.who.int/ipcs/assessment/public_health/fluoride/es/

Organización Mundial de la Salud (OMS) (2015); "Nota informativa sobre la ingesta de azúcares recomendada en la directriz de la OMS para adultos y niños"; Sitio Web

Oficial; Ginebra, Suiza. Disponible en: https://www.who.int/nutrition/publications/guidelines/sugar intake information no te es.pdf?ua=1

Organización Mundial de la Salud (OMS) (2012); "Salud Bucodental"; Sitio Web Oficial; Nota Informativa $\mathrm{N}^{\circ} 318$; Ginebra, Suiza. Disponible en: http://www.who.int/mediacentre/factsheets/fs318/es

Organización Mundial de la Salud. OMS. (1989). Vigilancia y evaluación de la Salud Bucodental. Informe de un Comité de Expertos. En: Serie de Informe Técnicos. Ginebra, Suiza. Páginas 29-30.

Raza Ximena, Alvear Ana, Andrade Ruth, Ayala Eugenia, Chilliquinga Mónica, Luque Ibelia, Pinto Galud, Pullas Amparito, Mendoza Susana, Sánchez Elisa (2011). "Estudio Epidemiológico Nacional de Salud Bucal en Escolares Menores de 15 años del Ecuador". Sitio Web Oficial de la Organización Panamericana de la Salud. Boletín Informativo. Edición N²9. Repositorio ISBN: 978-92-75-32028-0 Quito, Ecuador.

Roche Martínez Alina, Nasco Hidalgo Nayda, Gispert Abreu Estela de los A., Jiménez Echemendia Tania, Ventura Hernández Maria Isabel (2009). "Lesiones incipientes de caries dental y su relación con la higiene bucal en niños venezolanos". Rev Cubana Estomatología. Cuba, Dic [citado 2018 Ago 12] ; 46( 4 ): 70-89. Disponible en: http://scielo.sld.cu/scielo.php?script=sci_arttext\&pid=S0034$75072009000400008 \& \operatorname{lng}=\mathrm{es}$

Rowan-Legg Anne, Canadian Paediatric Society, Community Paediatrics Committee (2013). "Oral health care for children - a call for action". Paediatrics and Child Health. Volume 18. Issue 1. Canadá. 1 January. Pages: 37-43. Disponible en: https://doi.org/10.1093/pch/18.1.37

Sanchez Alberto, Childers, Fox, Bradley (1997). "Physicians' views on pediatric preventive 
dental care”. Revista Pediatric Dental Volumen 19 №3. Estados Unidos. Pag: 77383.

Scottish Intercollegiate Guidelines Network. SIGN (2014). "Dental interventions to prevent caries in children”. SIGN publication No. 138. Edinburgh, Reino Unido. Marzo. 138. Disponible en: https://www.sign.ac.uk/assets/sign138.pdf

Seif Tomás (1997). Cariología. Prevención, "Diagnóstico y Tratamiento Contemporáneo de la Caries Dental". Editorial Actualidades Médico Odontológicas Latinoamérica; primera edición; Venezuela; pag 13-33,35-55,179-215,241-333

Selwitz Robert, Amid Ismail, Nigel Pitts (2007). "Dental Caries". The Lancet. Volumen 369. Issue 9555. Estados Unidos. Pag: 51 - 59. ISSN 0140-6736. Disponible en: http://www.sciencedirect.com/science/article/pii/S0140673607600312

Tapias Ledesma, Jiménez-García, Lamas, Gil de Miguel (2002). "Efectividad de los selladores de fisuras en una población infantil con alto riesgo de presentar caries". Atención Primaria. Volume 30. Issue 3. Madrid. España. Pages 150-156. ISSN $0212-$ 6567.

en: http://www.sciencedirect.com/science/article/pii/S0212656702789956)

Tranaeus Sofia, Susan Al-Khateeb, Stein Björkman, Svante Twetman, Birgit AngmarMånsson (2001). "Application of quantitative light-induced fluorescence to monitor incipient lesions in caries-active children. A comparative study of remineralisation by fluoride varnish and professional cleaning". European Journal of Oral Sciences. Volumen 109. United States. December. Pages: 71-75. Disponible en: https://doi.org/10.1034/j.1600-0722.2001.00997.x

Viaña Francys, Belkis López, Mary Borges (2005). "Efectividad de sellantes de fosas y fisuras en la prevención de caries dental en molares sanos y con fluorosis en escolares. Período 1999-2004”. Odous Cientifica Volumen VI. No 1. Carabobo. Venezuela. Disponible en: http://servicio.bc.uc.edu.ve/odontologia/revista/v6n1/6-1-1.pdf

Wolff Mark Steven y Larson Charlie (2009). "The cariogenic dental biofilm: good, bad or just something to control?". Brazilian oral research 23 Suppl 1:31-8. New York, EEUU. Pag $32 . \quad$ Disponible en: https://www.researchgate.net/publication/38019697_The_cariogenic_dental_biofilm _Good_bad_or_just_something_to_control

Newman Michael, Takei Henry y Klokkevold Perry. "Periodontología Clínica de Carranza". Editorial Amolca. Volumen N 10. México: McGraw-Hill. 2010. Pag 170.

Zaror Carlos; Pineda Patricia; Villegas Mónica (2011). "Estudio clínico del primer molar permanente en niños de 6 años de edad de la comuna Calbuco, Chile". Acta Odontológica Venezolana. Volumen $49 \mathrm{~N}^{\circ} 3$. Chile.

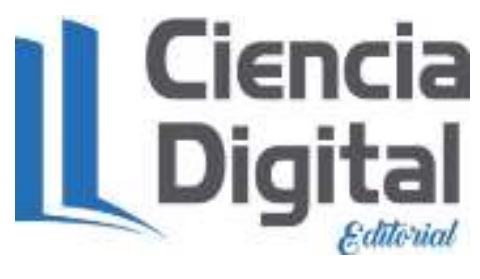




\section{Para citar el artículo indexado.}

Romero M., \& Romero H. (2019). Factores de protección de caries dental y su efectividad de aplicación, en los menores de 6 a 9 años: Resultados de estudio piloto. Revista electrónica Ciencia Digital 3(2), 77-92. Recuperado desde: http://cienciadigital.org/revistacienciadigital2/index.php/CienciaDigital/article/view/376/821

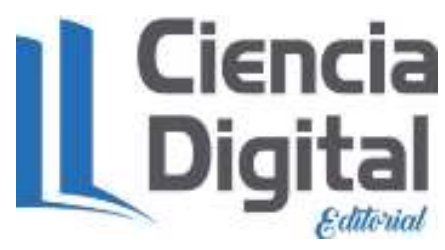

El artículo que se publica es de exclusiva responsabilidad de los autores y no necesariamente reflejan el pensamiento de la Revista Ciencia Digital.

El artículo queda en propiedad de la revista y, por tanto, su publicación parcial y/o total en otro medio tiene que ser autorizado por el director de la Revista Ciencia Digital.
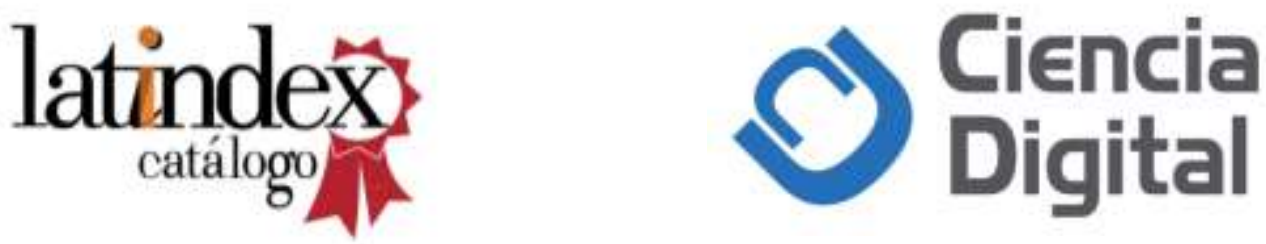